\title{
Study of Angiographic Anatomy of Right Coronary artery.
}

\author{
Dr. Jyoti Kulkarni ${ }^{1}$, Dr. Lopa Mehta ${ }^{2}$

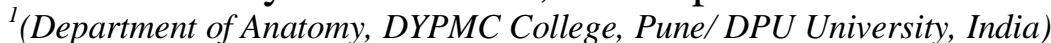 \\ ${ }^{2}$ (Department of Anatomy, GSMC College, Mumbai/ MumbaiUniversity, india)
}

\begin{abstract}
Coronary angiography is the radiographic visualisation of the coronary vessels after the injection of a radiopaque contrast media. The radiographic images are recorded for the future review with $35 \mathrm{~mm}$ cine film or digital recording. Percutaneous or cut down techniques, usually from the femoral or brachial artery, are used for the insertion of special intravascular catheters. Coronary angiography requires selective cannulation of the ostium of the left and right coronary arteries to obtain optimal selective contrast injection and imaging. The anatomy of coronary artery varies and several nomenclatures have been used to describe the anatomy and extent of disease. Currently, the most common one is that used in Coronary Artery Surgery Study (CASS), recently modified by the Bypass Angioplasty Revascularisation Investigation(BARI). In this tree the coronary nomenclature is divided into 29 segments with the ability to account for anatomic variations. In the present era people are opting for coronary angioplasty and coronary artery Bypass surgery almost on the counter in countries rich and poor. Knowledge of variations in the angiographic anatomy of coronary artery is vital in during interpretation of coronary angiography films, and managing congenital and acquired paediatric and adult heart diseases.
\end{abstract}

Keywords - Angiographic anatomy, Angioplasty, Bypass, Right coronary artery, ostium,.

\section{INTRODUCTION}

In the left anterior oblique view, the major axis outflow tract of the left ventricle and ascending aorta is not vertical, rather it tilts slightly to the left of the observer. Schematically, the outflow tract of the left ventricle and the aorta may be regarded as a nearly cylindrical structure with a bulge in the middle owing to the presence of aortic sinuses of the ascending aorta (Sino aortic plane or plane of aortic ring).The line drawn along the lower edge of this bulge seperates the floor of aortic cusps from the outflow tract of the left ventricle. These landmarks are easily identifiable in an aortogram and the origin of two coronary arteries can be described in relation to them ${ }^{1}$.

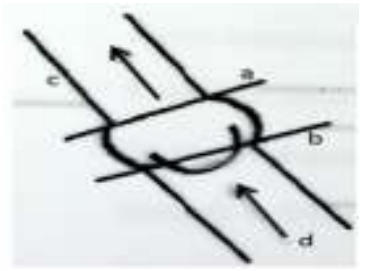

$$
\begin{aligned}
\text { Figure } & 1 . \\
\text { a- } & \text { Sinoaortic plane } \\
\text { b- } & \text { Aortoventricular plane } \\
\text { c- } & \text { Ascending aorta } \\
\text { d- } & \text { Left ventricular outflow tract }
\end{aligned}
$$

Right Coronary artery originates from the right sinus of the Valsalva and it is best seen and catheterised in the left anterior oblique view. After the right coronary artery has reached the acute margin of the heart, it then curves posteriorly and follows the posterior atrio ventricular groove towards the crux cordis. In left anterior oblique view this change of direction is seen as shallow with a smooth angle occasionally highlighted by the take off of the acute marginal branch. In the right anterior oblique view on the other hand this angle is more acute and the posterior and the anterior side of the angle may be superimposed. In order of their origin the branches of the right coronary artery are: Conus, Sinoatrial nodal branch, Right ventricular branch, Atrial branch, Acute marginal branch, branch to atrioventricular node, Posterior descending(Posterior intervenricular branch), branch to left ventricle, branch to left atrium. The conus is a fairly small vessel, which curves away from the main right coronary artery and proceeds ventrally, encircling the outflow tract of the right ventricle at about the level of pulmonary valve. In the right anterior oblique view, it appears directed to the right. The distal twigs of the branch may join a branch of the left coronary artery to form the anastomotic circle of Vieussens ${ }^{1,2}$, which is visible in occlusion of right coronary artery, or left anterior inteventricular artery which is an alternative source of blood supply to an area distal to the occlusion. Since the ostium is quite small its catheterisation is quite difficult. When the Sinoatrial node is the branch of right coronary artery, it usually originates from the initial portion of this vessel and runs in the direction opposite to that of the conus branch i.e cranially, dorsally and to the right. In its course along the anterolateral aspect of the atrioventricular groove, the right coronary artery gives origin to one or more anterior ventricular branches distributed to the wall of the right ventricle. These vessels may vary in length and number. In the right anterior oblique view these vessels seem to originate almost at right angles to the main artery. The acute marginal branch is relatively large and constant, 
and is directed towards the apex. In left anterior oblique view it departs from the margin and runs as a long straight vessel towards the bottom frame. The right atrial artery originates at about the level of the acute marginal branch but travels in opposite direction, cranially and towards the right border of heart. In its posterior portion between the acute marginal branch and the crux cordis it gives small branches to the posterior ventricular wall. In left anterior oblique view the right coronary artery makes a sharp upside down "U" curve and terminates into atrioventricular, posterior descending, left ventricular, left atrial branches. The best way to visualise the posterior descending is in the right anterior oblique view. It is distinguished by the fact that several short septals leave the posterior descending at $90^{\circ}$ angle. More than one branch originate from the right coronary artery distal to the crux and runs along the posterior aspect of the left ventricle supplying the diaphragmatic portion of the free left ventricular wall. These branches are best seen in $45^{0}$ left anterior oblique view ${ }^{1}$ (Figure 2).

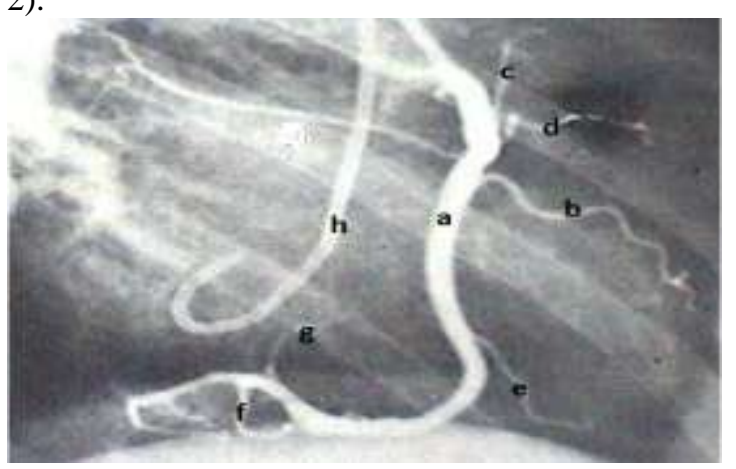

$$
\begin{aligned}
& \text { Figure } 2 \text { - Right anterior oblique } \\
& \text { view of right coronary system } \\
& \text { a - Right coronary artery } \\
& \text { b - Anterior ventricular branch } \\
& \text { c- Conus } \\
& \text { d- anterior ventricular branch } \\
& \text { e - Acute marginal branch } \\
& \text { f - Posterior interventricular } \\
& \text { branch } \\
& \text { g - Branch to AV node } \\
& \text { h - catheter }
\end{aligned}
$$

\section{AIMS AND OBJECTIVES}

In the present era people are opting for coronary angioplasty and coronary artery Bypass surgery almost on the counter in countries rich and poor. On reviewing the available literature, a wide range of variations in the coronary arteries was found. Hence the aim was to study the variations in the branching pattern of right coronary artery and its dominance pattern on cineangiographic films. To correlate the above studies and their clinical implication in Indian population.

\section{MATERIALS AND METHODS}

Observation on 107 consecutive antemortem coronary angiograms of random adult population in whom the coronary angiograms were indicated and were performed by the cardiologist at KEM Hospital, G.S Medical College, Mumbai. The dye used was Trazograph 76\% (Na-Diatrozate, an iodine based dye). Judkins catheter for right coronary artery was used. Catheterization was done by Seldingers technique, by percutaneous puncture of the femoral artery. The cardiologist performed the procedure in those patients in whom it was indicated. In this study parameters which could be measured with the available facilities were Variation in the origin of Right coronary artery, Variation in the branching pattern of Right coronary artery, Myocardial bridging and looping, Dominance pattern.

\section{OBSERVATIONS}

In one out of 107 cases the right coronary artery was found to originate from left posterior aortic sinus. It took a course anterior to aorta and continued in the right atrio ventricular groove (Figure 3).

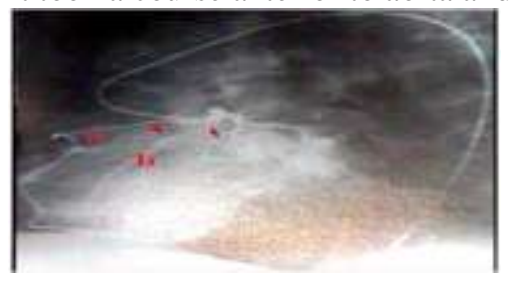

$$
\begin{aligned}
& \text { Figure } 3 \text { - Left anterior oblique view } \\
& \text { of left coronary system } \\
& \text { a - Right coronary artery originating } \\
& \text { from the left posterior aortic sinus } \\
& b \text { - Left anterior descending artery }
\end{aligned}
$$

In all the cases the right conus artery was found to be a branch of right coronary artery (Figure 2). In 84 out of 107 cases the right coronary artery showed preponderance over the left coronary artery (Figure 2). In 10 out of 107 cases the posterior descending artery was found to be a branch from both the right coronary artery as well as circumflex coronary artery.

In all the cases studied the branch to SA node was a branch from right coronary artery arising opposite to that of the conus.

When the sinoatrial node receives a branch from the left coronary artery, it often arises from the initial portion of the circumflex artery. It then ascends to the right beneath the left auricular appendage and behind the 
aorta crossing the posterior aspect of left atrium to reach the interatrial septum. It terminates around the base of the superior vena cava ${ }^{1}$.

\section{DISCUSSION}

- The right coronary artery was found to be dominant over the left in $79 \%$ of the cases.

- In present study in all cases the right conus was found to be a branch from the right coronary artery. However in $40 \%$ of cases where conus might have an independent origin, it has a very small accessory ostium in the anterior aortic sinus which may fail to get opaciifed during the angiography procedure ${ }^{\mathbf{8}}$.

- The right coronary artery usually arises from the anterior aortic sinus. In one out of 107 cases it was found to have ectopic origin from the left posterior aortic sinus. However its prevalence was observed to be $0.0008 \%$ as mentioned by Yamanaka and Hobbs ${ }^{3}$. Simkoff WL in 1982 reported a case of anomalous origin of Right coronary artery from the Left anterior descending artery. Multiple attempts to cannulate this artery were unsuccessful and an aortic flush injection confirmed its absence ${ }^{4}$. Incidence of origin of right coronary artery is reported to be $0.13 \%$ by Shin-Min Yuan et al, and this anomaly is increasingly reported with newer study techniques. The patients with this anomaly may remain asymptomatic ${ }^{9}$.

- The discovery of coronary artery variations in patients undergoing coronary angiography is about $0.6 \%$ to $1 \%{ }^{3}$.

- Coronary endothelial sprouts occur at around the $5^{\text {th }}$ week of intra uterine life from the bulbus cordis which has not yet differentiated into the aorta and the pulmonary trunk. The first evidence of coronary vessel development is the appearance at the beginning of the fifth week of structure like blood islands just under the epicardium in the sulci of the developing heart ${ }^{2}$.

- Coronary vascular endothelial maturation closely parallels the development of the embryonic epicardium. The first coronary endothelial cells migrate from the region of the developing liver and establish contact with the sinus venosus. Vascular precursors cells and small vessels then invade the sinus venosus wall, ventricular and atrial myocardium, and mesenchymal border of the aortic annulus. Initially multiple connections between the coronary vascular plexus and the aortic myocardium are present; however only two connections persist. Coronary endothelial cells do not participate in the formation of the intramyocardial vascular net work. The orderly development of the coronary arterial branching pattern and elastic lamina is dependent upon the neural crest cells. The maturation of coronary vasculature represents both angiogenesis and vasculogenesis. It is suggested that the developing capillary vessels in the sulci induce the sprouting of coronary veins and arteries $\mathbf{5 , 6}$.

- According to the theory of Ogden J. there is a dual origin of coronary arteries - Proximal and distal. The distal portion develops first. It is comprised of a retiform vascular network, similar to the capillary network that forms in the other parts of body. This network develops in the interventricular and atrioventricular grooves and forms a complete ring around the developing vessels (Peritruncal ring), and communicates with the heart chamber and the extracardiac graet vessels. Furthur development of some vessels and regression of others; the final coronary pattern develops. This theory adequetly explains the variations of coronary vasculature ${ }^{7}$.

- Knowledge of variations in the angiographic anatomy of coronary artery is vital during interpretation of coronary angiography films, and managing congenital and acquired paediatric and adult heart diseases.

\section{REFERENCES}

[1] Gensini, Goffredo G.; Coronary Arteriography by Goffredo G. Gensini, Ch-9, "Arterial Anastomosis in coronary circulation”, Pg 163, New York, Futura Publishing Company, 1975.

[2] Decker, G.A.G; duPleissis, D.J.; Lee Mcgregor's Synopsis Of Surgical Anatomy, $12^{\text {th }}$ edition, Ch-22 "The heart and the great vessels", Pg - 280; K.M. Vargheese Company, Dadar, Bombay 400014, India, 1986.

[3] Bergman, Ronald A.; Afifi, Adel K.; Miyauchi Ryosuke; Illustrated Encyclopedia Of Human Anatomic Variation,Coronary arteries Pg 2-18.

[4] Simkoff, W.L., Murphy,E.S.,Demots H, Khousaris, Addruzzese P; Anomalous origin of right coronary artery from the left anterior descending; Angiographic diagnosis in a patient with coronary artery disease; Catheterisation and Cardiovascular diagnosis 1982;8:49-53.

[5] Larsen, William J.; Larsen's Human Embryology, Ch-7 “Development of Vasculature”, Pg-191; New York, Edinburgh, Churchill Livingstone, 1993.

[6] Keller, Bradly B., Markwald, Rodger R.; Hurst's The Heart, $9^{\text {th }}$ Edition ch-8 “Embryology of Heart” Pg -195 ; New York, Mc Graw Hill, Health Professions Division 1998.

[7] Ogden J: The origin of the Coronary arteries: Circulation 1968(38): suppl 6:150.

[8] Gensini Goffredo G.; Coronary arteriography by Goffredo G. Gensini, Ch-8 “Angiographic anatomy of coronary arteries”, Pg-132, New York, Futura Publishing Company, 1975

[9] Shin-Min Yuan, MD; Salis tager,MD;Ehud Raanani MD; Anomalous origin of right coronary artery from the left coronary sinus; Chang Gung Med J Vol.32 No.4, July - Aug 2009. 